\title{
论 文
}

\section{外界刺激对钠通道基因突变的窦房结体系起搏 功能的调控作用}

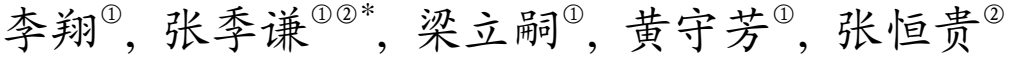 \\ (1) 安徽师范大学物理与电子信息学院, 芜湖 241000; \\ (2) Biological Physics Group, School of Physics \& Astronomy, The University of Manchester, Manchester, M139PL, UK \\ * 联系人, E-mail: zhangcdc@mail.ahnu.edu.cn
}

收稿日期: 2013-03-20; 接受日期: 2013-05-07

国家自然科学基金(批准号：11047017)、威康信托基金(批准号：081808/Z/06/Z)、英国生物工艺生物科学研究基金(批准号：BBS/B1678X) 和安徽省高等学校省级优秀青年人才基金项目(批准号: 2011SQRL023)资助

\begin{abstract}
摘要采用完整的二维兔子窦房结心房细胞模型, 考虑 2 种类型钠离子基因突变的影响, 如功能缺失型突变、功能获得型突变, 本研究考察了包括迷走神经分泌的乙酰胆碱、酸性浓 度和组织温度 3 种外界刺激对心脏起搏和传导能力的调控作用. 研究结果表明, 这 2 种类型 的基因突变对起搏速率、传导能力有不同的影响. 本研究还发现, 这些突变的消极作用可通 过 3 种外界刺激进行调节: 增加乙酰胆碱浓度可增强突变诱导窦房结心搏徐缓的效果; 改变 酸浓度或调节组织温度可以有效地增强窦房结驱动周围心房组织及恢复组织起搏行为的能 力. 上述研究将有助于揭示钠离子通道疾病的内在机制, 并有望提供一些可能的治疗手段.
\end{abstract}

关键词

基因突变

计算机模拟

钠通道

异常起搏

二维窦房结模型
众所周知，离子通道结构和正常功能表达是细胞 生理活动的基础, 特定位置的基因突变会导致通道的 激活和失活, 引起组织功能性紊乱或遗传性疾病 ${ }^{[1]}$. 有 研究工作者发现, 基因突变的研究将可为东南亚国家 的人群预防和治疗症疾提供重要的线索 ${ }^{[2]}$. 人们发现编 码钠离子通道的 SCN $5 A$ 基因突变会引起其蛋白质结构 和功能的异常, 从而诱发一系列心律异常类疾病 ${ }^{[3]}$.

研究发现, 有 3 种类型的离子通道突变, 如功能 缺失型突变 (loss of function mutation, LFM) 、功能获 得型突变 (gain of function mutation, GFM) 及无功能 型突变(non-functional mutations, NFM). 其中, LFM 会减少钠离子电流并加速通道的失活过程. GFM 则
会增加钠离子电流并延缓通道的失活，该通道持续 开放并延缓了钠离子电流的增加过程, 导致动作电 位时程的增加. LFM 会引起 Brugada 综合征 ${ }^{[46]}$ 、病态 窦房结综合征 ${ }^{[7,8]}$ 、进行性家族心脏传导阻滞 I 型 (progressive familial heart block type I $)^{[9]}$. GFM 将会诱 导长 QT 综合征 ${ }^{[10,11]}$ 、婴儿猝死综合征(sudden infant death syndrome $)^{[12,13]}$. SCN5A 基因突变还可以引起复 合性的临床体征, 人们称之为心脏钠离子通道重叠综 合征 ${ }^{[14]}$. 然而, 到目前为止, 还没有充足的证据表明, 第三种类型的突变会对离子通道的功能产生影响.

现有实验表明对前 2 种类型的 SCN $5 A$ 基因突变 的研究仍停留在分子水平. 在近期研究发现, 环境扰

引用格式: 李翔, 张季谦, 梁立嗣, 等. 外界刺激对钠通道基因突变的突房结体系起搏功能的调控作用. 中国科学: 生命科学, 2013,43: 657-662

英文版见: Zhang J Q, Li X, Liang L S, et al. Effects of external stimuli on the pacemaker function of the sinoatrial node in sodium channel gene mutations models. Sci China Life Sci, 2013, 56, doi: 10.1007/s11427-013-4533-x 
动对细胞内离子动力学过程有积极的调控作用 ${ }^{[15,16]}$, 因此,很值得思考这样的问题：这些因基因突变所引 起的钠离子通道缺陷是否能够改善? 利用外界刺激 是否可以提高窦房结的起搏行为和传导性能, 甚至 是从异常状态恢复至正常水平? 这些是值得人们进 一步研究的具有挑战性的课题.

因此, 本研究中, 以完整的兔子窦房结-心房细 胞二维模型为研究对象, 考虑钠离子通道 2 种类型基 因突变的影响, 重点研究了外界刺激对起搏行为和 传导过程的调控作用.

\section{1 模型描述及方法}

为研究具有基因突变的心脏起搏活动的离子机 制, 采用兔子心脏窦房结及心房细胞的动作电位模 型, 其满足的动力学方程如下 ${ }^{[17,18]}$ :

$$
\begin{aligned}
C_{\mathrm{m}} \frac{\mathrm{d} V}{\mathrm{~d} t}= & -\left(I_{0}+I_{\mathrm{Na}}+I_{\mathrm{CaL}}+I_{\mathrm{CaT}}+I_{\mathrm{K}, \mathrm{ACh}}\right)=-I_{\mathrm{tot}}, \\
I_{0} & =I_{\mathrm{NaK}}+I_{\mathrm{NaCa}}+I_{\mathrm{K}}+I_{\mathrm{to}}+I_{\mathrm{st}}+I_{\mathrm{f}},
\end{aligned}
$$

其中, $V$ 和 $C_{\mathrm{m}}$ 表示膜电压和膜电容(单位 $\mu \mathrm{F}$ ), $I_{\mathrm{Na}}$ 是 TTX-敏感性的钠离子电流, $I_{\mathrm{CaL}}$ 和 $I_{\mathrm{K}, \mathrm{ACh}}$ 分别表示 $\mathrm{L}$ 型 $\mathrm{Ca}^{2+}$ 通道电流和乙酰胆碱活化的钾离子通道电流, $I_{0}$ 是细胞中其他所有电流总和. 该模型中各方程中电 流及相关参数的详细描述可参见文献 $[17,18]$.

由于心脏起搏活动是一个非常复杂的集体行为, 为研究外界刺激对其带来的影响, 在该模型中, 将中 心窦房结、边缘窦房结和心房等细胞作为节点构建一 个 $375 \times 45$ 的二维耦合网络结构, 每个细胞满足上述 方程(1). 该网络模型满足如下方程 ${ }^{[18]}$ :

$$
C_{\mathrm{m}}(i, j) \frac{\partial V(i, j)}{\partial t}=-I_{\mathrm{tot}}+\nabla \cdot[D(i, j) \nabla V(i, j)],
$$

其中, $(i, j)$ 标注细胞的空间位置坐标, $D(i, j)$ 表征通过 间隙连接扩散耦合的膜电压的扩散系数, $\nabla$ 描述细 胞中的空间梯度算符.

本研究中, 主要探讨两个问题: (i) 钠离子通道 中 2 种类型基因突变(LFM 和 GFM)对窦房结起搏功能 的影响; (ii) 3 种外界刺激对心脏起搏能力的调控作 用. 模拟过程中, 时间和空间步长分别设为 $0.005 \mathrm{~s}$ 和 $0.04 \mathrm{~mm}$. 采用有限差分方法对上述方程进行数值模 拟, 将分布在二维模型中部的细胞动作电位记录并 加以处理, 计算出细胞膜电压的周期间隔并进行相关 分析和讨论.

\section{2 结果与讨论}

本实验室研究 2 种类型突变对窦房结起搏行为 的影响. 这 2 种类型突变都会导致钠离子通道功能的 异常表达. 模型中对应的钠离子电流满足如下方程:

$$
I_{\mathrm{Na}}=\left(k \cdot g_{\mathrm{Na}}\right) m^{3} h[\mathrm{Na}]_{0}^{+} \frac{V F^{2}}{R T} \frac{\mathrm{e}^{\left(V-E_{\mathrm{Na}}\right) F / R T}-1}{\mathrm{e}^{V F / R T}-1},
$$

其中, $g_{\mathrm{Na}}$ 是钠离子通道的电导, $m$ 和 $h$ 是激活和失活 门控变量. 在该模型中, 基因突变的影响可用参数 $k$ 描述, 即, 当 $k<1$ 时, 钠电流将减少, 这表示产生了 LFM 现象; 而当 $k>1$ 时, 引起钠电流增加, 表明产生 GFM 现象 ${ }^{[19]}$. 式(2)和(3)中各个电流的详细描述和物 理含义可参见文献[17 19].

图 1 给出了 2 类基因突变对窦房结起搏能力影 响的模拟结果. 从图中可以看出, 与正常的搏动速 率相比 (wild type: WT, 正常起搏周期 $C L=274.2 \mathrm{~ms}$ ), LFM 会降低边缘窦房结细胞的起搏速率(图 1C), 而 对中心窦房结没有影响(图 1A). 同样地, GFM 会增 加边缘窦房结细胞的起搏速率(图 1D), 对中心窦房 结也没有影响(图 1B) $)^{[19,20]}$. 然而, 在二维耦合模型 中, 若不考虑乙酰胆碱的作用, LFM 将会减慢搏动 速率, 导致窦性搏动过缓甚至窦性停搏(图 $1 \mathrm{E}, k=0.6$, 起搏周期 $C L=297.9 \mathrm{~ms}$ ); 而 GFM 则将会加快搏动速 率, 导致窦性过速和房颤等症状(图 $1 \mathrm{~F}, k=1.4, C L=$ $257.5 \mathrm{~ms})$.

\section{1 外界刺激信号对迷走神经的作用}

为研究迷走神经分泌的乙酰胆碱对有基因突变 的窦房结起搏行为的影响, 本研究选取乙酰胆碱及对 其敏感相关离子电流(如 $I_{\mathrm{K}, \mathrm{ACh}}, I_{\mathrm{Ca}, \mathrm{L}}$ ) 作为调控参数 ${ }^{[17]}$. 这些电流的动力学过程满足如下方程:

$$
\begin{aligned}
& I_{\mathrm{K}, \mathrm{ACh}}= g_{\mathrm{K}, \mathrm{ACh}}\left(\frac{\left[\mathrm{K}^{+}\right]_{\mathrm{e}}}{10+\left[\mathrm{K}^{+}\right]_{\mathrm{e}}}\right) \\
& \times\left(\frac{V_{\mathrm{m}}-E_{\mathrm{K}}}{1+\exp \left[\left(V_{\mathrm{m}}-E_{\mathrm{K}}-140\right) F / 2.5 R T\right]}\right), \\
& I_{\mathrm{Ca}, \mathrm{L}}=g_{\mathrm{Ca}, \mathrm{L}}\left[f_{\mathrm{L}} d_{\mathrm{L}}+\frac{0.006}{\left.1+\mathrm{e}^{-(V+144.1) / 6}\right]\left(V-E_{\mathrm{Ca}, \mathrm{L}}\right)(1-b),}\right. \\
& g_{\mathrm{K}, \mathrm{ACh}}=g_{\mathrm{K}, \mathrm{ACh}, \max j j} j \frac{[\mathrm{ACh}]^{n_{\mathrm{K}, \mathrm{ACh}}}}{K_{0.5, \mathrm{~S}, \mathrm{~K}, \mathrm{ACh}}^{n_{\mathrm{K} h}}+[\mathrm{ACh}]^{n_{\mathrm{K}, \mathrm{ACh}}}},
\end{aligned}
$$

其中, $g_{\mathrm{K}, \mathrm{ACh}}$ 和 $g_{\mathrm{Ca}, \mathrm{L}}$ 表示通道电导, $g_{\mathrm{K}, \mathrm{ACh}, \max }$ 是电导 



图 1 基因突变对窦房结博动功能的影响

LFM (左侧), GFM (右侧). 中心窦房结细胞(A 和 B), 边缘窦房结细胞 (C 和 D), 整个组织细胞(E 和 F), 其中 SAc, SAp, AM 分别表示窦房结中 心区域(central SAN)、窦房结边缘区域(peripheral SAN)和心房(atrial muscle)

$g_{\mathrm{K}, \mathrm{ACh}}$ 的最大值. $j$ 和 $k$ 是失活变量, [ACh]表示乙酰胆 碱浓度. 式(4)中各量的详细描述请参见文献[17 19].

本研究将模型记录线上从窦房结到心房各个细 胞的动作电位时空演化过程描述在图 2 中. 从图中可 以看出，当没有外界刺激作用于体系，即 $[\mathrm{ACh}]=0.0$ 时, 由中心区域的窦房结细胞所产生的动作电位信 号, 依次传导至边缘窦房结细胞和心房细胞区域(图
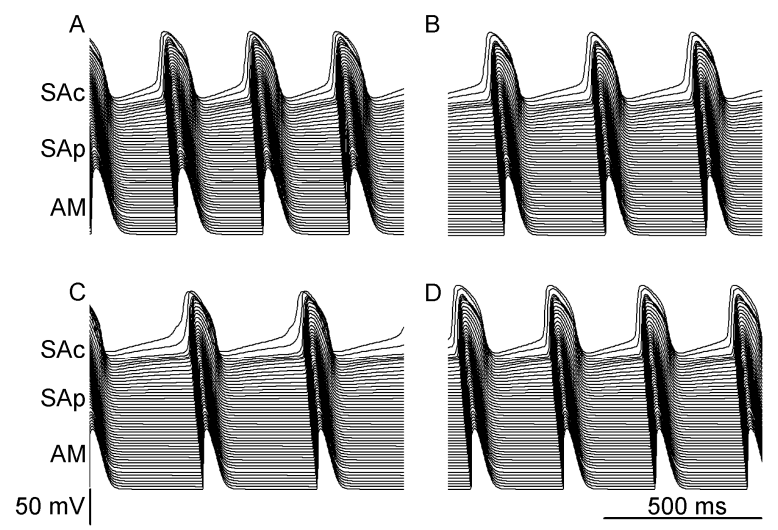

图 $2[\mathrm{ACh}]$ 对窦房结搏动功能的影响

A: 正常组织且无 ACh 分泌情形, $C L=274.2 \mathrm{~ms} ; \mathrm{B}, \mathrm{C}, \mathrm{D}$ 中 $[\mathrm{ACh}]=1.5 \times 10^{-8} \mathrm{~mol} / \mathrm{L} ; \mathrm{B}$ : 正常组织, $C L=321.5 \mathrm{~ms}$; C: LFM ( $\left.k=0.6\right)$, $C L=363 \mathrm{~ms}$; D: GFM ( $k=1.4), C L=293 \mathrm{~ms}$
$2 \mathrm{~A}$ ); 而当 $[\mathrm{ACh}]$ 增加至 $1.5 \times 10^{-8} \mathrm{~mol} / \mathrm{L}$ ，搏动周期 $C L$ 将会从正常的 $274.2 \mathrm{~ms}$ 增大到 $321.5 \mathrm{~ms}$ (图 2B), 这 表明乙酰胆碱对窦房结体系具有负作用效果. 在图 2B 条件下, 引入基因突变的影响发现, 这会进一步 放大体系不正常起搏行为, 并且会呈现不同的特征. 例如, 对于具有 LFM 的体系 $(k=0.6)$, 搏动周期会增 大至 $363 \mathrm{~ms}$ (图 2C). 进一步地, 如果 [ACh]增大到 $5.0 \times 10^{-8} \mathrm{~mol} / \mathrm{L}$, 窦房结的起搏活动会进一步降低, 严重的甚至会引起窦性停搏. 而对于 GFM 体系 $(k=1.4)$, 搏动周期会减小到 $293 \mathrm{~ms}$ (图 2D). 其中有 关 LFM 的模拟结果与临床上所观察到的现象相吻合. 例如, 人们可以观察到夜晚睡觉后人体窦房结中乙 酰胆碱的浓度较白天高些，这将会导致心脏节律的 变缓甚至诱发心脏猝死 ${ }^{[6,21]}$.

\section{2 利用药物调节体系的酸性浓度}

最近实验结果表明, $\mathrm{T}$ 型䥻离子通道电流 $I_{\mathrm{Ca}, \mathrm{T}}$ 对 体系中的酸碱浓度较为敏感 ${ }^{[22]}$. 酸性较高的环境会 阻滞 $\mathrm{Ca}^{2+}$ 的运输和降低心肌细胞中酶的活性, 从而 妨碍心脏肾上腺的作用, 导致心肌收缩能力的降低 和肌无力. 因此, 为减少基因突变的消极作用, 提高 窦房结的起搏能力, 可通过调节 $I_{\mathrm{Ca}, \mathrm{T}}$ 的电导参数 $\alpha$ 来 
实现，该电流满足方程:

$$
I_{\mathrm{Ca}, \mathrm{T}}=\alpha \cdot g_{\mathrm{Ca}, \mathrm{T}} \cdot d_{\mathrm{T}} \cdot f_{\mathrm{T}} \cdot\left(V-E_{\mathrm{Ca}, \mathrm{T}}\right),
$$

模拟中, 固定 $[\mathrm{ACh}]=1.5 \times 10^{-8} \mathrm{~mol} / \mathrm{L}$, 选择电导 参数 $\alpha$ 作为可调参量. 图 3 给出了存在基因突变情况 下，体系起搏行为随酸浓度变化关系的示意图. 从图 $3 \mathrm{~A}$ 中可以看出, 对于 LFM 突变 $(k=0.6)$, 当设置 $\alpha$ 从 1.0 变化到 1.7 时(图中箭头表示当 $t>3.0 \mathrm{~s}$ 调节作用的


表明酸浓度有重要的调节效果. 进一步发现, 如果将 $[\mathrm{ACh}]$ 增大到引起心脏死振的浓度水平时 $\left(5.0 \times 10^{-8}\right.$ $\mathrm{mol} / \mathrm{L}$ )，可以通过将参数 $\alpha$ 增加到 3.5 而恢复体系的 正常振荡行为. 同样地, 对于 GFM突变 $(k=1.4)$, 如果 通过将参数 $\alpha$ 从 1.0 降低到 0.2 , 就可将搏动周期 $C L$ 从 $293.4 \mathrm{~ms}$ 调节到正常水平. 模拟结果如图 3B 所示.

\section{3 调节体系的温度}

最近的研究报告表明, 体温对心率有一定的调 节作用，并且这种作用是直接作用于窦房结而并不 是神经系统 ${ }^{[23]}$. 例如, 用 $41^{\circ} \mathrm{C}$ 的温水浸浴 $10 \mathrm{~min}$ 或 进行 $15 \mathrm{~min} 60^{\circ} \mathrm{C}$ 的桑拿浴, 均可使慢性心衰者心率 每分钟增加 20 25 次 ${ }^{[22] ;} 15 \mathrm{~min}, 37^{\circ} \mathrm{C}$ 热水浴使受试 者的心输出量升高, 这与心率增快有关 ${ }^{[24,25]}$. 研究发 现，窦房结的去极化速率主要由电流 $I_{\mathrm{f}}, I_{\mathrm{k}}$ 和 $I_{\mathrm{Ca}, \mathrm{T}}$ 来决 定. 其中 $I_{\mathrm{f}}$ 在窦房结起搏速率中起着十分重要的作用, 该电流满足方程 ${ }^{[26]}$ :

$$
I_{\mathrm{f}}=\lambda \cdot\left[g_{\mathrm{f}, \mathrm{Na}} \cdot y \cdot\left(V-E_{\mathrm{Na}}\right)+g_{\mathrm{f}, \mathrm{K}} \cdot y\left(V-E_{\mathrm{K}}\right)\right],
$$

值得一提的是, 电流 $I_{\mathrm{f}}$ 的传导率参数 $\lambda$ 会随着组 织温度的升高而增大，因此可以通过设置该参数来 反映温度作用效果，从而实现对搏动速率的调节. 模 拟过程中, 固定乙酰胆碱浓度 $[\mathrm{ACh}]=1.5 \times 10^{-8} \mathrm{~mol} / \mathrm{L}$.
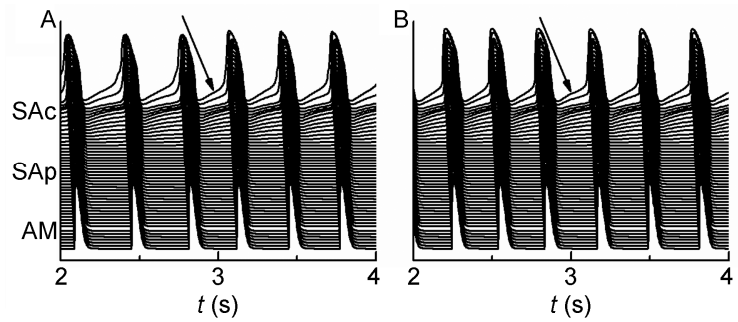

图 $3 \mathrm{pH}$ 对窦房结搏动功能的影响

$[\mathrm{ACh}]=1.5 \times 10^{-8} \mathrm{~mol} / \mathrm{L}$. A: LFM, $k=0.6$, 当 $t<3.0 \mathrm{~s}$ 时, $\alpha=1.0, C L=363$ $\mathrm{ms}$; 当 $t>3.0 \mathrm{~s}$ 时, $\alpha=1.7, C L=324.9 \mathrm{~ms}$; B: GFM, $k=1.4$, 当 $t<3.0 \mathrm{~s}$ 时, $\alpha=1.0, C L=293.4 \mathrm{~ms}$; 当 $t>3.0 \mathrm{~s}$ 时, $\alpha=0.2, C L=322.8 \mathrm{~ms}$. 图中箭头 表示调节 $\alpha$ 的时间点
结果如图 4 所示. 从图中可以看出，当 $\lambda=1.0$ 时，对于 LFM ( $k=0.4)$, 搏动周期达到 $C L=673.3 \mathrm{~ms}$ ，这种情形 下很容易诱发窦性停搏(图 4A, $t<3.0 \mathrm{~s}$ ). 而对于 GFM 突变 $(k=2.0)$, 搏动周期 $C L$ 减少到 $266 \mathrm{~ms}$, 这又会引 起赎性过速和房颤(图 4B, $t<3.0 \mathrm{~s}$ ). 此时,可以通过调 节参数 $\lambda$ 到适当值来消除这 2 种异常的搏动行为. 例如, 对于 LFM 可以增大 $\lambda$ 到 1.4, 而对于 GFM 则减少 $\lambda$ 到 0.6. 最后，研究了体系在不同突变种类 $(k$ 不同 $)$ 及不 同外界刺激 $(\lambda$ 不同)的条件下，体系搏动行为的变化 规律. 图 5 是心脏起搏状态随温度参数 $\lambda$ 和突变 $k$ 变 化的灰度示意图. 图中横坐标是基因突变参数 $k$, 纵 坐标是外界刺激温度参量 $\lambda$. 灰度的明亮程度表示体 系搏动能力的大小. 从图中可以看出, 随着突变参数 $k$ 的变化, 体系的重新起搏能力随外界刺激变化而表 现出不同的特性. 据此, 可将图形划分为 I , II， III 和 IV 这四个区域. 在区域 I, 当 $\lambda>1.0$ 并且 $k>0.8$ 时, 窦房结能够自发且持续地振荡. 由于起搏周期 $C L<$ $250 \mathrm{~ms}$ ，处于此区域中的细胞通常表现为心动过速状 态; 当参数 $k$ 和 $\lambda$ 处于区域III中时, $330 \mathrm{~ms}<C L<450 \mathrm{~ms}$, 表明体系处于心搏徐缓状态; 在区域IV中, $C L>450 \mathrm{~ms}$, 体系处于死振区域，在该区域中，无论用何种刺激均 无法使体系恢复正常振动状态. 然而, 处在区域 II 中 的体系不同于处在区域 I，III和IV中的情形，体系不 需要任何外界刺激即可工作于正常模式. 图 5 结果表 明，除了区域IV以外，体系的搏动活性可以利用外界 适当的刺激加以恢复。例如，在区域 I 和III中，尽管 因基因突变所诱发的异常搏动或甚至心脏猝死是常 见的且容易促发的病症现象，它们却可以通过上述 3 种方法之一来激活恢复. 例如，在区域 I 中，当 $\lambda>1.0$ 并且 $k>0.8$, 窦房结可以自发且持续的振荡, 当然,

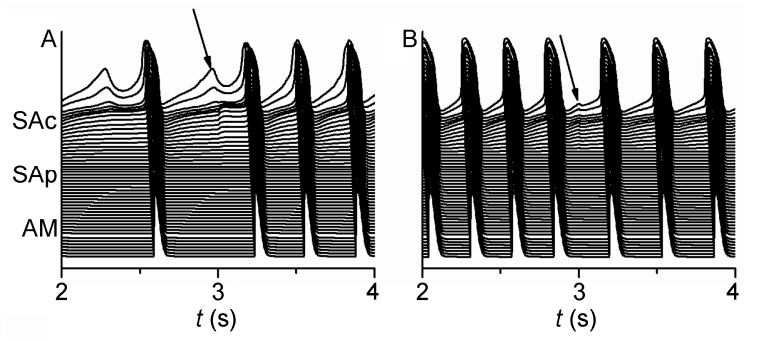

图 4 体系温度对窦房结搏动功能的影响 $[\mathrm{ACh}]=1.5 \times 10^{-8} \mathrm{~mol} / \mathrm{L}, \lambda=1.0$. 图中箭头表示 $3 \mathrm{~s}$ 后开始调节体系温 度的时间点. A: LFM, $k=0.4, \lambda$ 从 1.0 增大到 $1.4, C L$ 则从 $673.3 \mathrm{~ms}$ 降 低到正常值 $329 \mathrm{~ms} ; \mathrm{B}: \mathrm{GFM}, k=2.0, \lambda$ 从 1.0 减小到 $0.6, C L$ 则从 $266 \mathrm{~ms}$ 增加到正常值 




图 5 心脏搏动状态随温度参数 $\lambda$ 和突变 $k$ 变化的灰度 示意图

整个参数区分为四个不同的区域. I：心动过速区域 $(C L<250 \mathrm{~ms})$; II : 正常节律区域 $(250 \mathrm{~ms}<C L<330 \mathrm{~ms})$; III：心动过缓区域 (330 ms $<C L<450 \mathrm{~ms}) ; \mathrm{IV}$ : 死振区域 $(C L>450 \mathrm{~ms})$

由于起搏周期 $C L<250 \mathrm{~ms}$, 体系主要工作在 GFM 区间 $(k>1.0)$, 因此, 心脏通常处在心动过速状态, 在这种情 形下, 若引入外界刺激( $\lambda$ 从 1.0 减小到 0.6$), C L$ 将会从 $266 \mathrm{~ms}$ 增加到正常的 $329 \mathrm{~ms}$, 这时, 心脏就可以完全复 苏到正常起搏活动状态 (图 4B). 同样地, 也可以用类似 的方法将处于区域III中的体系恢复到正常状态.

\section{3 结论}

本研究中, 采用兔子的二维心脏窦房结一心房解 剖模型，考虑存在 2 种类型基因突变的情形下，研究 了外界不同刺激对体系起搏能力的影响. 研究发现, 一方面, 对于存在 GFM 突变的有缺陷的心脏体系会 诱发心动过速, 而存在 LFM 突变缺陷则会引起心动 过缓, 严重时甚至会导致临床上所观察到的心脏猝 死现象. 另一方面, 值得注意的是, 模拟结果表明, 上述不正常的起搏甚至死振状态可以通过适当的外 界刺激来减轻甚至消除.

上述模拟结果对于临床治疗有一定的指导意义. 可以通过调整不同的控制参数, 诸如 $k, \lambda$ 和酸性浓 度来调控体系的起搏行为. 当然这种调节作用只是 在一定的参数范围内才起作用(图 5 中的区域 I, II, III). 如果超出了该参数区间, 特别是在存在 LFM 突 变情形下 $(k<0.3$, 图 5 中区域 $I V)$, 无论用什么刺激 均无法恢复体系的正常状态. 本研究的模拟结果将 为临床上心律不齐选取适当治疗方案提供一种有益 的参考.

\section{参考文献}

1 Heribert S, Konig I R, Sekar K, et al. Large-scale association analysis identifies 13 new susceptibility loci for coronary artery disease. Nature Genet, 2011, 43: 333-338

2 杨银峰, 朱月春, 李丹怡, 等. 中国西南阿昌族 G6PD 缺陷的特征及新单体型 $487 \mathrm{G}>\mathrm{A} / \mathrm{IVS} 5-612(\mathrm{G}>\mathrm{C})$ 的鉴定. 中国科学 $\mathrm{C}$ 辑: 生命 科学, 2007, 37: 460-465

3 Herfst L J, Book M B, Jongsma H J. Trafficking and functional expression of cardiac Na ${ }^{+}$channels. J Mod Cell Cardiol, 2004, 36: 185-193

4 Shimizu W, Aiba T, Kamakura S. Mechanisms of disease: current understanding and future challenges in Brugada syndrome. Nat Clin Pract Cardiovasc Med, 2005, 2: 408-414

5 Juang J M, Huang S K. Brugada syndrome-an under-recognized electrical disease in patients with sudden cardiac death. Cardiology, 2004, 101: $157-169$

6 Vatta M, Dumaine R, Varghese G, et al. Genetic and biophysical basis of sudden unexplained nocturnal death syndrome (SUNDS), a disease allelic to Brugada syndrome. Hum Mol Genet, 2002, 11: 337-345

7 Benson D W, Wang D W, Dyment M, et al. Congenital sick sinus syndrome caused by recessicve mutations in the cardiac sodium channel gene (SCN5A). J Clin Invest, 2003, 112: 1019-1028

8 Dobrzynski H, Boyeet M R, Anderson R H. Newo insights into pacemaker activity: Promoting understanding of sick sinus syndrome. Circulation, 2007, 115: 1921-1932

9 Schott J J, Alshinawi C, Kyndt F, et al. Cardiac conduction defects associated with mutations in SCN5A. Nature Genet, 1999, 23: 20-21

10 Chiang C E. Congenital and acquired long QT syndrome: current concepts and management. Cardiol Rev, 2004, 12: 222-234

11 Towbin J A, Vatta M. Molecular biology and the prolonged QT syndromes. Am J Med, 2001, 110: 385-398

12 Ackerman M J, Siu B L, Sturner W Q, et al. Postmortem molecular analysis of SCN5A defects in sudden infant death syndrome. JAMA, 2001, 286: 2264-2269

13 Plant L D, Bowers P N, Liu Q, et al. A common cardiac sodium channel variant associated with sudden infant death in African Americans, SCN5A S1103Y. J Clin Invest, 2006, 116: 430-435 
14 Viswanathan P C, Balser J R. Inherited sodium channelopathies: a continuum of channel dysfunction. Trends Cardiovasc Med, 2004, 14: $28-35$

15 Chen H S, Zhang J Q, Liu J Q. Selective effects of external noise on $\mathrm{Ca}^{2+}$ signal in mesoscopic scale biochemical cell systems. Biophys Chem, 2007, 125: 397-402

16 Zhang J Q, Qi F, Xin H W. Effects of noise on the off rate of $\mathrm{Ca}^{2+}$ binding proteins in a coupled biochemical cell system. Biophys Chem, 2001, 94: 201-207

17 Zhang H, Holden A V, Kodama I, et al. Mathematical models of action potentials in the periphery and center of the rabbit sinoatrial node. Am J Physiol Heart Circ Physiol, 2000, 279: 397-421

18 Zhang H, Zhao Y, Lei M, et al. Computational evaluation of the roles of $\mathrm{Na}^{+}$current, $\mathrm{i}_{\mathrm{Na}}$, and cell death in cardiac pacemaking and driving. Am J Physiol Heart Circ Physiol, 2007, 292: 165-174

19 Timothy D B, Oleg VA, Shin I, et al. Mechanistic links between $\mathrm{Na}^{+}$channel (SCN5A) mutations and impaired cardiac pacemaking in sick sinus syndrome. Circ. Res, 2010, 107: 126-137

20 Honjo H, Boyett M R, Kodama I, et al. Correlation between electrical activity and the size of rabbit sinoatrial node cells. J Physiol, 1996, 496: 795-808

21 Wani B A, Khalil M Z, Al-Nozha M M, et al. Aborted sudden nocturnal cardiac death in a young man with structurally normal heart. Saudi Med J, 2005, 26: 323-325

22 Brian P D, Jonathan S. pH modification of human T-type calcium channel gating. Biophys J, 2000, 78: 1895-1905

23 Pakhcmov A G, Mathur S P, Doyle J, et al. Comparative effects of extremely high power microwave pulses and brief CW irradiation on pacemaker function in isolated frog heart slices. Bioelectromagnetics, 2000, 110: 351-360

24 Tei C, Horikiri Y, Park J C, et al. Acute hemodynamic improvement by thermal vasodilation in congestive heart failure. Circulation, 1995, 91: 2582-2590

25 Boone T, Westendorf T, Ayres P, Cardiovascular responses to a hot tub bath. J Altern Complem Med, 1999, 5: 301-304

26 Rosenbaum T, Gordon S E. Quickening the pace: looking into the heart of HCN channels. Neuron, 2004, 42: 193-196 